\title{
Effects of Rotational Energy Relaxation in a Modular Particle-Continuum Method
}

\author{
Timothy R. Deschenes, $\stackrel{*}{-}$ Timothy D. Holman,, \pm and Iain D. Boyd \\ University of Michigan, Ann Arbor, Michigan 48109
}

DOI: $\underline{10.2514 / 1.50720}$

\begin{abstract}
A modular particle-continuum method is extended to include thermal nonequilibrium between translational and rotational energy modes to simulate hypersonic steady-state flows that exhibit small regions of collisional nonequilibrium in a mainly continuum flowfield. This method loosely couples an existing direct-simulation Monte Carlo code to a Navier-Stokes solver (computational fluid dynamics) while allowing both time step and cell size to be completely decoupled between each method. By limiting the size of the direct-simulation Monte Carlo region to only areas in collisional nonequilibrium, the modular particle-continuum method is able to reproduce full direct-simulation Monte Carlo results for flows with global Knudsen numbers of 0.01 and 0.002 while decreasing the computational time required by factors of 2.94 and 28.1, respectively. The goal of the present study is to include consistent models that separate rotational and translational modes in both flow modules. Inclusion of rotational relaxation decreases the computational cost of the modular particle-continuum method.
\end{abstract}

\section{Nomenclature}

$C_{h}=$ heat transfer coefficient

$C_{p}=$ pressure coefficient

$C_{\tau}=$ shear stress coefficient

$d \quad=$ diameter, $\mathrm{m}$

$f$

$g$

$k$

Presented as Paper 2010-0808 at the 48th AIAA Aerospace Sciences Meeting, Orlando, FL, 4-7 January 2010; received 11 May 2010; revision received 4 October 2010; accepted for publication 8 December 2010. Copyright $(2010$ by Timothy R. Deschenes, Timothy D. Holman, and Iain D. Boyd. Published by the American Institute of Aeronautics and Astronautics, Inc., with permission. Copies of this paper may be made for personal or internal use, on condition that the copier pay the $\$ 10.00$ per-copy fee to the Copyright Clearance Center, Inc., 222 Rosewood Drive, Danvers, MA 01923; include the code $0887-8722 / 11$ and $\$ 10.00$ in correspondence with the CCC.

*Graduate Student, Department of Aerospace Engineering; thytimo@ umich.edu. Student Member AIAA.

Graduate Student, Department of Aerospace Engineering; tholman@ umich.edu. Student Member AIAA.

†Professor, Department of Aerospace Engineering; iainboyd@umich.edu. Associate Fellow AIAA.

$$
\begin{array}{lll}
\text { Subscripts } & \\
\text { ROT } & =\text { rotation } \\
\text { ref } & =\text { reference } \\
s & = & \text { species } \\
\text { TRA } & = & \text { translation } \\
W & = & \text { wall } \\
\infty & = & \text { freestream }
\end{array}
$$

\section{Introduction}

A S A hypersonic vehicle enters a planetary atmosphere, the variation of characteristic flow length and time scales about the vehicle leads to a multiscale gas flow. One parameter used to compare the variation in length scales is the Knudsen number. At sufficiently low Knudsen numbers, many gas particle collisions occur around the body and the flow can be considered in collisional equilibrium such that the velocity distribution function can be described by a small perturbation from the equilibrium velocity distribution function. When the flow is considered near collisional equilibrium, continuum flow formulations such as the Navier-Stokes equations provide a physically accurate description of the flowfield. Computational fluid dynamics (CFD) methods provide accurate and efficient numerical solutions to the Navier-Stokes equations. However, at high Knudsen numbers, the flow can be considered rarefied, or in collisional nonequilibrium. In this regime, the approximations used to derive the Navier-Stokes equations break down, and the flow can only be accurately described using a kinetic description. The directsimulation Monte Carlo (DSMC) method, first proposed by Bird [1], provides an approach that can accurately model dilute gas flows described by the Boltzmann equation. Although physically accurate over all flow conditions, the DSMC method becomes computationally intensive at higher densities due to time and length scale restrictions set by separating the move and collision processes. At these higher densities, the approximations used to form the NavierStokes equations are valid, and the flow can be computed using CFD. For many hypersonic flows of interest, some regions of the flowfield, such as the shock, boundary layer, and near-wake region, can be sufficiently rarefied so that CFD cannot be used over the entire flowfield while simultaneously having regions that can be considered well within the continuum regime making full DSMC simulations prohibitively expensive. Instead, a hybrid method can be employed that takes advantage of the computational efficiency of CFD in regions that are near collisional equilibrium, while maintaining the physical accuracy of the DSMC in regions that are rarefied.

In addition to containing regions displaying continuum breakdown, many hypersonic flows have regions in which the 
rotational energy mode is not in equilibrium with the translational modes. Although regions that are rarefied also show this thermal nonequilibrium, other regions of the flow, such as the flow directly behind a strong shock, can be considered near collisional equilibrium while the difference between translational and rotational temperatures is still significant. To decrease the size of the DSMC region, which increases the numerical efficiency of the MPC method, a rotational relaxation model that is consistent with the rarefied module is implemented within the continuum module of the hybrid method.

Previous work has been performed using a zonally decoupled DSMC-CFD simulation method [2-4]. For these methods, a CFD solution is calculated to a predetermined interface, then this information is used as the boundary condition for the DSMC simulation over the remainder of the domain. This method is only valid when the rarefied region is completely downstream of the continuum region with no recirculation occurring across the interface. This is because the CFD solution is completely decoupled from the DSMC solution.

Often, rarefied regions are highly localized, and two way coupling is required to obtain an efficient, physically accurate simulation. Various methods have been proposed that adaptively reposition the interface between DSMC and CFD throughout the simulation using different coupling methods between the two domains [5-9]. Reference [10] presents a discussion of the major considerations involved in coupling a kinetic scheme with a continuum method, as well as a summary of published work on past methods. Recently, Schwartzentruber and Boyd have described a modular particle-continuum (MPC) method that was first developed for one-dimensional (1-D) shock waves [11] and later extended to two-dimensional (2-D) and axisymmetric flows $[12,13]$. By limiting the DSMC method to only regions that are rarefied, the MPC method can achieve speedup factors exceeding three for transitional flows [14] and 13 for near-continuum flows [13].

In addition to continuum breakdown, past hybrid methods applied to hypersonic flows have included continuum solvers that are incapable of modeling the thermal nonequilibrium between translational and rotational energy modes. To still maintain the physical accuracy of the hybrid solver, regions in which thermal nonequilibrium existed were automatically added to the DSMC domain regardless of continuum breakdown $[9,12,15]$. Although the results remained physically accurate, the additional breakdown parameter increased the computational cost of the hybrid method. Instead, by modeling the rotational relaxation in the continuum solver, this region can be accurately modeled at a reduced computational cost.

The present study extends the MPC method to include a rotational nonequilibrium model in the continuum solver that is consistent with the rarefied relaxation model [16] used in the DSMC module. By including a rotational nonequilibrium model within the $\mathrm{CFD}$ module, the regions that can be solved with CFD can be expanded due to the increase in physical accuracy of the CFD module [17,18]. This, in turn, decreases the size of the DSMC region, which leads to an overall decrease in the computational expense of the MPC method. The elements of the MPC method, including the rotational relaxation models used in each flow module, the selection of the interface location between flow solvers, and information transfer procedures between flow modules, are described in Sec. II. Section III describes the flow conditions for the two cases used to examine the effects of rotational nonequilibrium with the MPC method, while Sec. IV compares full DSMC, full CFD, and MPC predictions of macroscopic flowfield quantities and surface properties and the computational expense of the MPC method relative to full DSMC. Finally, conclusions drawn from these simulations are presented in Sec. $\underline{\text { V. }}$

\section{Hybrid Method}

The MPC code used in this study was developed to be capable of simulating 1-D shock waves [11] and both axisymmetric and 2-D steady-state hypersonic flows $[\underline{12}, 19]$. It uses a CFD code, LeMANS
$[20,21]$, for the continuum regions, while using the DSMC code, MONACO [22], to simulate the rarefied regions.

\section{A. Flow Modules}

LeMANS is capable of simulating 2-D, axisymmetric, or threedimensional (3-D) steady-state continuum, hypersonic flows using a finite volume formulation of the Navier-Stokes equations, with the added ability to account for vibrational and rotational nonequilibrium. The inviscid fluxes are solved using a modified form of the Steger-Warming flux vector splitting method, which is less dissipative and can be used to calculate boundary layers. The method switches back to the original form of Steger-Warming near shock waves. The viscous terms are calculated using properties at cell nodes and cell centers. The no-slip condition is applied to wall boundaries, while the inflow and outflow are treated as supersonic flow. Time integration is performed using a point-implicit method. It can also simulate multispecies and weakly ionized flows with finite rate chemistry, but those models are not used in this study. The variable hard sphere (VHS) model is used to calculate the viscositytemperature relation that can be formulated using Eqs. (1) and (2), where $\omega, \mu_{\text {ref }}$, and $T_{\text {ref }}$ are constants, and $d_{\text {ref }}$ is the species reference diameter [1]. All results presented within this paper simulate molecular nitrogen with a reference diameter of $d_{\text {ref }}=4.17 \times$ $10^{-10} \mathrm{~m}$ at $T_{\text {ref }}=273 \mathrm{~K}$ with a power law exponent of $\omega=0.75$ :

$$
\begin{gathered}
\mu=\mu_{\text {ref }}\left(\frac{T_{\mathrm{TRA}}}{T_{\text {ref }}}\right)^{\omega} \\
\mu_{\text {ref }}=\frac{15 \sqrt{\pi m k T_{\text {ref }}}}{2 \pi d_{\text {ref }}^{2}(5-2 \omega)(7-2 \omega)}
\end{gathered}
$$

MONACO is a general cell-based implementation of the DSMC method capable of simulating rotational and vibrational nonequilibrium and multispecies flow with finite rate chemistry. The VHS collision model that replicates the macroscopic viscositytemperature dependence modeled in LeMANS is used. The following subsections describe and compare the internal relaxation processes modeled within both the CFD and DSMC modules.

\section{B. Rotational Relaxation}

Both the CFD and DSMC modules have the capability of simulating rotational nonequilibrium [23]. For the CFD module, a separate rotational energy equation is added to the total system of equations solved. Equation (3) shows the time rate of change of rotational energy per unit volume, where $\mathbf{q}_{\text {ROT }}$ is the rotational heat flux given by Fourier's law, $\mathbf{u}_{d s}$ is the species diffusion velocity given by Fick's law, and $\dot{w}_{\mathrm{ROT}}$ is the rotational energy source term:

$$
\begin{gathered}
\frac{\partial \rho e_{\mathrm{ROT}}}{\partial t}+\nabla \cdot\left(\rho e_{\mathrm{ROT}} \mathbf{u}\right)=-\nabla \cdot\left(\mathbf{q}_{\mathrm{ROT}}\right)-\nabla \\
\cdot \sum_{s}\left(\rho_{s} e_{\mathrm{ROT}, s} \mathbf{u}_{d s}\right)+\dot{w}_{\mathrm{ROT}} \\
\dot{w}_{\mathrm{ROT}}=\sum_{s}\left(S_{\mathrm{ROT}, s}^{\mathrm{TRA}-\mathrm{ROT}}+\dot{w}_{s} e_{\mathrm{ROT}, s}\right)
\end{gathered}
$$

Equation (4) shows that the rotational source term can be split into two parts: a rotation-translation relaxation source and a source due to the gain and loss of rotational energy through chemical reactions. For this study of a single species gas, the chemistry source is always zero. The rotation-translation relaxation source can be written as Eq. (5), where $e_{\mathrm{ROT}, s}^{*}$ is the specific rotational energy based on the transiational temperature, $e_{\mathrm{ROT}, s}$ is the current specific rotational energy, $Z_{\mathrm{ROT}, s}$ is the rotational collision number, and $\tau_{\mathrm{coll}, s}$ is the mean collision time. The rotational collision number, shown in Eq. (6), is derived from Parker [24]. The mean collision frequency, which is the inverse of the mean collision time, for the VHS model given by Bird [1] is shown in Eq. (7): 


$$
\begin{gathered}
S_{\mathrm{ROT}, s}^{\mathrm{TRA}-\mathrm{ROT}}=\rho_{s} \frac{e_{\mathrm{ROT}, s}^{*}-e_{\mathrm{ROT}, s}}{\tau_{\mathrm{ROT}, s}}=\rho_{s} \frac{e_{\mathrm{ROT}, s}^{*}-e_{\mathrm{ROT}, s}}{Z_{\mathrm{ROT}, s} \tau_{\mathrm{coll}}} \\
Z_{\mathrm{ROT}, s}=\frac{Z_{\mathrm{ROT}, s}^{\infty}}{1+\left(\pi^{3 / 2} / 2\right)\left(T_{s}^{*} / T_{\mathrm{TRA}}\right)^{1 / 2}+\left[\left(\pi^{2} / 4\right)+\pi\right]\left(T_{s}^{*} / T_{\mathrm{TRA}}\right)}
\end{gathered}
$$

$$
v_{s}=\frac{1}{\tau_{\mathrm{coll}, s}}=\sum_{i}\left(n_{i} d_{\mathrm{ref}, s, i}^{2}\left(\frac{8 \pi k T_{\mathrm{ref}, i}}{\mu_{s, i}^{*}}\right)^{1 / 2}\left(\frac{T_{\mathrm{TRA}}}{T_{\mathrm{ref}, i}}\right)^{1-\omega_{i}}\right)
$$

MONACO uses the variable rotational energy exchange probability model of Boyd [16], which is also based on Parker [24]. This formulation uses a probability of a rotationally inelastic collision $\phi_{\text {ROT } . s}\left(\varepsilon_{c}\right)$ that reproduces the macroscopic relaxation time by satisfying Eq. ( 8 ), where $\varepsilon_{c}$ is the total collisional energy between the two particles, and $f\left(\varepsilon_{c}\right)$ is the collisional energy probability distribution function. Lumpkin et al. [25] found that the definitions of continuum and particle relaxation times differ by a factor given by Eq. (9), where $\eta_{\text {int,TRA }}$ is the number of internal degrees of freedom based on the translational temperature, and $\omega$ is the VHS temperature exponent. Figure 1 compares the predicted temperatures of an adiabatic rotational relaxation process from both CFD and DSMC. Despite differences in how relaxation is modeled, the two models are consistent at the continuum limit, which results in excellent agreement:

$$
\begin{gathered}
\bar{P}_{\mathrm{ROT}, s}=\frac{1}{Z_{\mathrm{ROT}, s}}=\frac{1}{v_{s} \tau_{\mathrm{ROT}, s}}=\int_{0}^{\infty} \phi_{\mathrm{ROT}, s}\left(\varepsilon_{c}\right) f\left(\varepsilon_{c}\right) \mathrm{d}\left(\varepsilon_{c}\right) \\
\tau_{\mathrm{int}}^{\mathrm{DSMC}}=\frac{\tau_{\mathrm{int}}^{\mathrm{CFD}}}{1+\left[\eta_{\mathrm{int}, \mathrm{TRA}} /(4-2 \omega)\right]}
\end{gathered}
$$

\section{Interface Location and Continuum Breakdown}

Both the accuracy and efficiency of a hybrid DSMC-CFD method depend strongly on proper placement of the interface location. For physical accuracy, the interface must be located within regions that can be considered in collisional equilibrium, where the velocity distribution is only slightly perturbed from equilibrium and the Navier-Stokes equations are valid. To maximize efficiency, a hybrid method requires the interface between CFD and DSMC to be located near the edge of the collisional equilibrium region. The MPC method uses a gradient-length Knudsen number, first proposed by Boyd et al. [26] and Wang and Boyd [27]. Equation (10) shows the gradientlength Knudsen number, where $\lambda$ is the local mean free path, and $Q$ is some flow quantity of interest. For this work, gradient-length

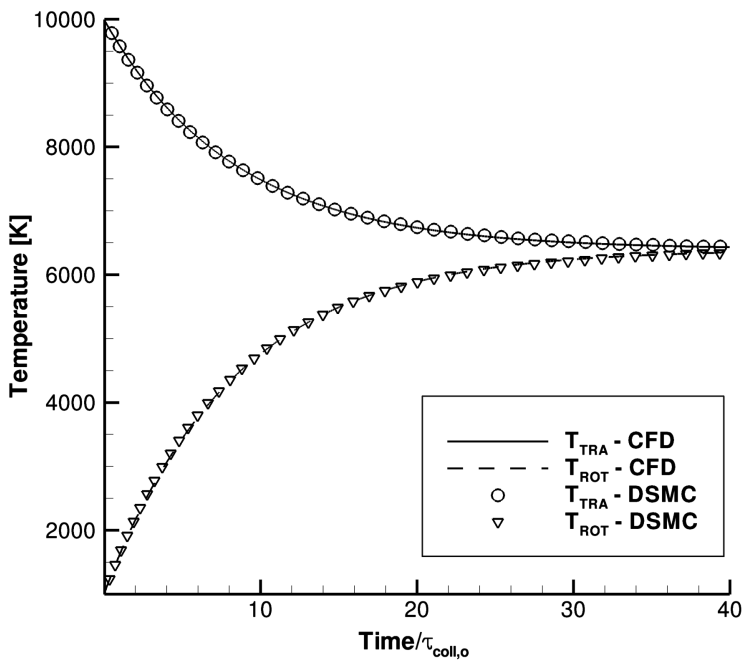

Fig. 1 Comparison of adiabatic rotational-translational relaxation processes predicted by DSMC and CFD.
Knudsen numbers based on density, velocity magnitude, translational temperature, and rotational temperature are used:

$$
K n_{\mathrm{GL}-Q}=\lambda\left|\frac{\nabla Q}{Q}\right|
$$

Previous research has shown, for hypersonic reentry problems $[12,28]$ and 1-D normal shock waves [11], that regions where the maximum gradient-length Knudsen number remains less than 0.05 display less than a $5 \%$ difference in flow quantities predicted by DSMC and CFD assuming perfect gas physics. Therefore, regions where the maximum gradient-length Knudsen number exceeds 0.05 are simulated with DSMC to maintain physical accuracy within the solution.

In addition to ensuring that the velocity distribution function is near equilibrium at the interface, the rotational energy distribution must also be near equilibrium. This is required so that the distribution of internal energies assigned to particles in DSMC boundary cells and relaxation rates calculated in both flow solvers remain consistent. In some regions of the flow, the velocity distribution functions may be very near equilibrium, while the rotational energy distribution function requires more collisions to reach equilibrium. To ensure that the rotational energy distribution function is near equilibrium, an additional breakdown parameter must be applied that characterizes the degree that the rotational energy distribution function departs from equilibrium. The largest cause of the departure of the rotational energy distribution function from the Boltzmann form is in regions where the flow is highly collisional and strong thermal relaxation takes place, such as the flow directly behind the bow shock in hypersonic flow, where the density and translational energy are large, but rotational relaxation is still significant. To characterize this process, the magnitude of the energy transferred by rotationtranslation relaxation processes is compared with the total amount of energy stored in rotational energy, which results in a thermal breakdown parameter seen in Eq. (11). This added breakdown parameter is of the same form suggested by Schwartzentruber et al., as described in [12], but is further relaxed to take into account the capability of calculating rotational relaxation processes in the continuum solver:

$$
K n_{\mathrm{ROT}-\mathrm{NEQ}}=\frac{\left|T_{\mathrm{TRA}}-T_{\mathrm{ROT}}\right|}{2 T_{\mathrm{ROT}}}
$$

Figure 2 shows a comparison of translational temperature prediction of the MPC method with (top) and without (bottom) the rotational nonequilibrium switch activated along with full DSMC. Without the nonequilibrium switch, the interface location between CFD and DSMC modules is located very near the shock. Since CFD does not contain the physical accuracy required to model this portion of the rotational relaxation process, as the rotational energy distribution function is highly non-Boltzmann in this region, the postshock temperatures are overpredicted compared with full DSMC. With a breakdown parameter of 0.05 , such that DSMC is used in regions where the difference between translational and rotational temperature exceeds $10 \%$ of the rotational temperature, the portion of the flowfield modeled with the continuum solver is slightly decreased to only regions where the continuum solver is physically valid, and agreement between MPC and full DSMC results is greatly improved. Velocity and rotational energy distribution functions are sampled from the full DSMC solution at locations at the edge of the interface location predicted by each MPC simulation, denoted as A and $\mathbf{B}$ in Fig. 2. Figures $\underline{3 a}$ and $\underline{3 b}$, respectively, compare the velocity distribution functions and rotational energy distribution function sampled from DSMC with equilibrium theory at point A. Although the velocity probability density functions are in excellent agreement with equilibrium theory, the rotational energy distribution function differs significantly from equilibrium. The peak in rotational energy probability density function at low rotational energies signifies that there is still a large number of particles that have not experienced a rotationally inelastic collision at the postshock temperature and still maintain the slope of an equilibrium probability density function at the freestream temperature. In contrast, Figs. $4 \mathrm{a}$ and $4 \mathrm{~b}$ compare the 


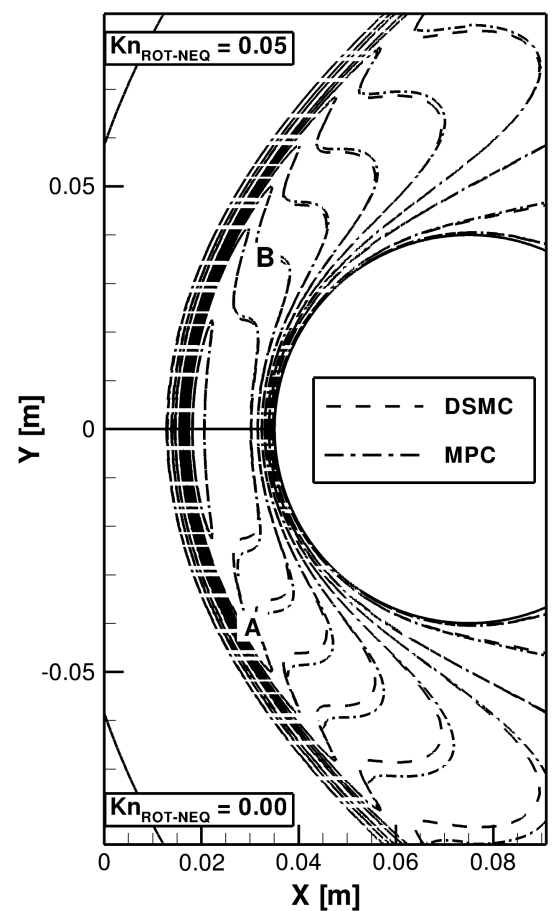

Fig. 2 Comparison of translational temperatures predicted by the MPC method with (top) and without (bottom) the rotational nonequilibrium breakdown parameter compared with full DSMC of Mach 12 flow over a cylinder with a global Knudsen number of 0.01 .

velocity and rotational energy distribution functions, respectively, at the continuum interface location computed with the added breakdown parameter at point $\mathbf{B}$. At this point in the flow, both the velocity and rotational energy probability density functions are in much better agreement with the equilibrium description, and the models used in the continuum solver are valid. Since the equilibrium rotational energy distribution function is calculated based on the average rotational energy, comparison of higher-order moments, such as the variance (calculated from sampled data and the equilibrium distribution), can be used as a measure of degree of rotational nonequilibrium. At point $\mathbf{A}$, the sampled variance differs by nearly $25 \%$ from equilibrium, while the sampled variance differs by less than $10 \%$ from the equilibrium value at point $\mathbf{B}$.

By applying a breakdown parameter that ensures that the energy distribution function is near equilibrium, internal energies assigned to particles in DSMC boundary cells are in excellent agreement with the rotational energies predicted in a full DSMC simulation. This ensures that the particles generated in the MPC method from the energy distribution function at the interface are consistent with the full DSMC simulation. This results in a final breakdown parameter, shown in Eq. (12):

$$
B r=\max \left(K n_{\mathrm{GL}-\rho}, K n_{\mathrm{GL}-T_{\mathrm{TRA}}}, K n_{\mathrm{GL}-T_{\mathrm{ROT}}}, K n_{\mathrm{GL}-|V|}, K n_{\mathrm{ROT}-\mathrm{NEQ}}\right)
$$

Figure 5 shows the final interface locations between rarefied and continuum solvers for the two flow conditions that are studied in this paper. For flow with a global Knudsen of 0.01 , a large portion of the flowfield, such as the thick bow shock, boundary layer, and wake region, is rarefied. In contrast, only the near-wake and thin boundary layers are rarefied at the lower Knudsen number. Even though the interior of the bow shock is highly nonequilibrium at any density, previous work [12] has shown that using DSMC in this region is not required for accurate prediction of flowfield properties behind the shock.

\section{Information Transfer}

The modular implementation of the MPC method allows both DSMC and CFD modules to maintain their own mesh and data structures. Information is transferred between each method using a state-based coupling procedure. The state-based coupling procedure assigns boundary cells on the edge of each module domain and transfers flow information, such as density, velocity components, and temperatures, to these cells from corresponding cells of the other module. Figure 6 shows a schematic of how data are transferred between the two modules at an interface location.

First, the interface location is found using the breakdown parameter shown in Eq. (12). Next, DSMC overlap cells are extended to create a buffer region. This buffer region is used throughout the unsteady portion of the simulation as the MPC method relaxes the initial CFD solution to reproduce the full DSMC result. To obtain an accurate DSMC prediction in rarefied regions, the buffer region must be large enough to eliminate any error caused by the initially inaccurate DSMC boundary conditions from the CFD module. For this work, the buffer region is at least 20 mean free paths, which ensures that a sufficient number of collisions occur to eliminate any error from the initial CFD solution propagating into the regions considered rarefied. Periodically, the breakdown parameter is reevaluated to ensure that the entire rarefied region is contained within the DSMC domain. Once the interfaces have stopped moving, information from the DSMC region is passed to the CFD edges, and the CFD module iterates. Particles are generated in DSMC boundary cells using macroscopic averages and gradients calculated from the
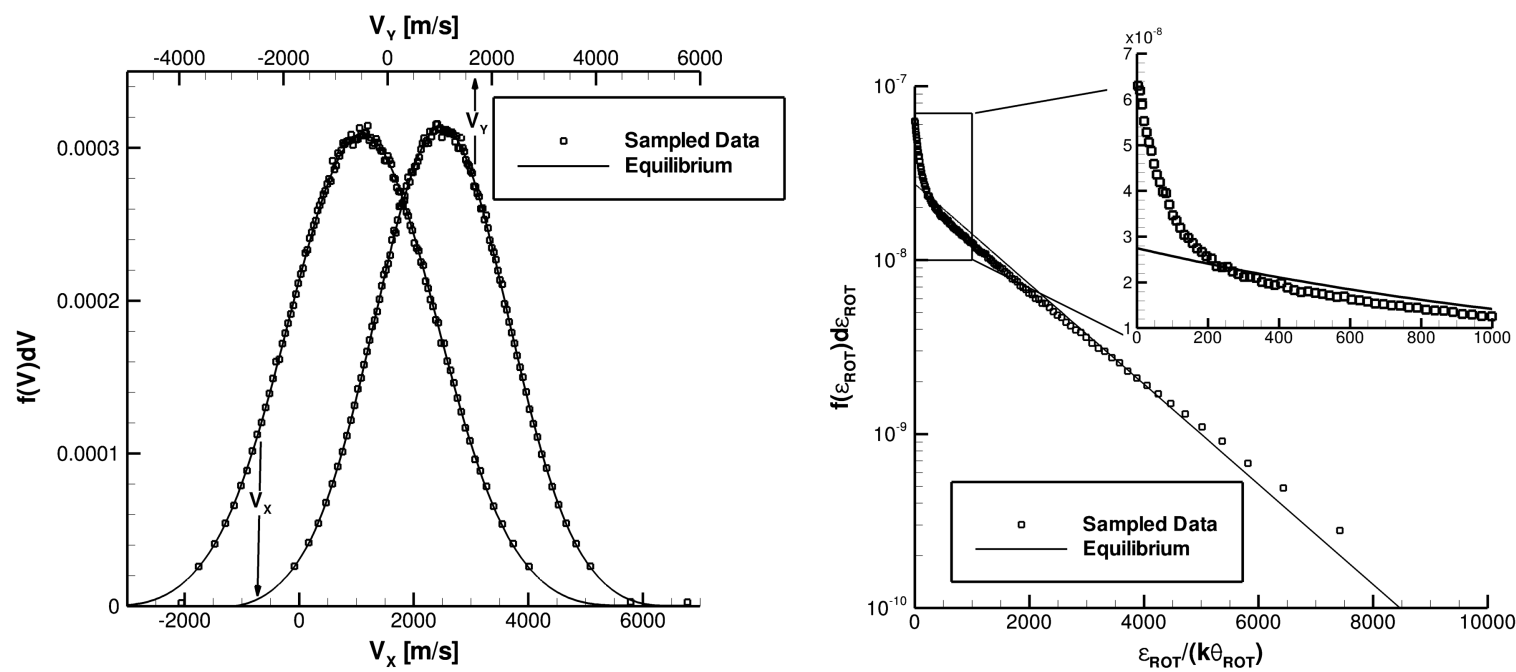

a) Velocity probability density functions

b) Rotational energy probability density function

Fig. 3 Comparison of probability density functions predicted by DSMC and equilibrium theory at point A, shown in Fig. 2. 


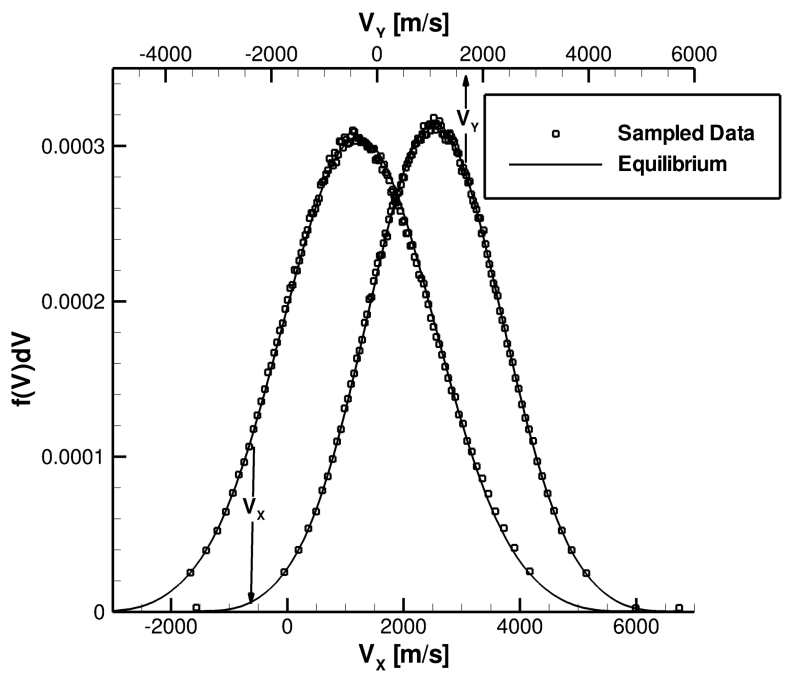

a) Velocity probability density functions

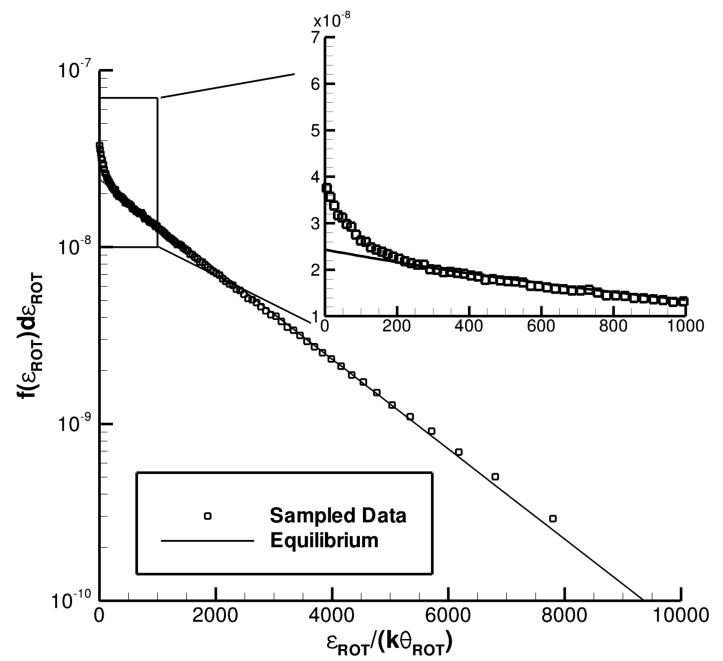

b) Rotational energy probability density function

Fig. 4 Comparison of probability density functions predicted by DSMC and equilibrium theory at point B, shown in Fig. 2.

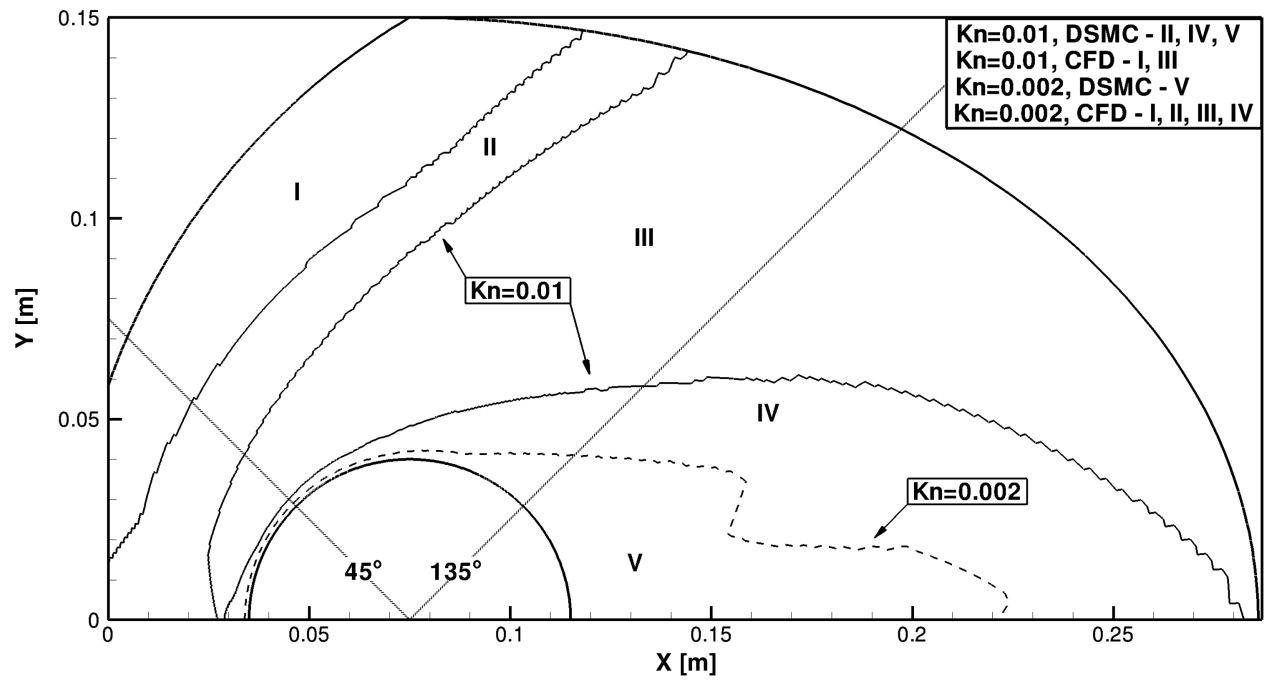

Fig. 5 Comparison of the final continuum-rarefied interface locations for Mach 12 flow over a cylinder at global Knudsen numbers of 0.002 and 0.01.

corresponding continuum cell. Particle velocities are sampled from the Chapman-Enskog velocity distribution function by an acceptance-rejection technique suggested by Garcia and Alder [29]. Rotational energies are assigned to each particle by sampling the rotational energy distribution function by inverse transform sampling. In CFD boundary cells, particle information is sampled in corresponding DSMC cells, and a subrelaxation average, shown in Eq. (13), is applied to reduce the statistical scatter in information collected from DSMC [30]:

$$
\bar{Q}_{j}=(1-\Phi) \bar{Q}_{j-1}+\Phi Q_{j}
$$

This performs a weighted average that applies a small weight to the current sample while applying a larger weight to the previous average. The MPC method then periodically switches between the two modules while allowing information to be transferred, adaptively moving the interface location at each module switch as the entire flowfield iterates to the steady-state solution.

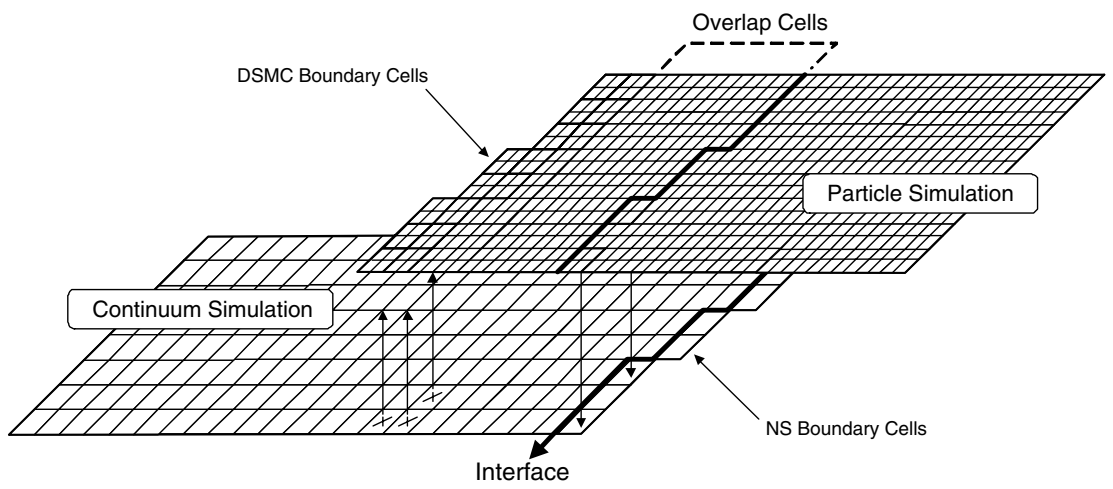

Fig. 6 Hybrid particle-continuum coupling procedure [14]. 


\section{Flow Conditions}

The implementation of rotational relaxation in the MPC method is evaluated with simulation of hypersonic flow of molecular nitrogen about a cylinder with a freestream Mach number of 12 at two Knudsen numbers. The freestream temperature is $T_{\mathrm{TRA}, \infty}=$ $T_{\text {ROT }, \infty}=217.5 \mathrm{~K}$ at a freestream velocity of $u_{\infty}=3,608 \mathrm{~ms}^{-1}$. The cylinder wall temperature is set to $T_{W}=1000 \mathrm{~K}$ with full diffuse reflection in DSMC simulations and a no-slip isothermal condition in CFD simulations. The freestream density is set to two different values of $\rho_{\infty}=7.48 \times 10^{-5} \mathrm{~kg} \mathrm{~m}^{-3}$ and $\rho_{\infty}=3.74 \times 10^{-4} \mathrm{~kg} \mathrm{~m}^{-3}$, which correspond to global Knudsen numbers based on cylinder diameters of $K n_{\infty}=0.01$ and $K n_{\infty}=0.002$, respectively. The diameter of the cylinder simulated is $d=8 \mathrm{~cm}$. Constant time steps of $1.5 \times 10^{-8} \mathrm{~s}$ and $4.0 \times 10^{-9} \mathrm{~s}$ are used in the DSMC method at global Knudsen numbers of $K n_{\infty}=0.01$ and $K n_{\infty}=0.002$, respectively, while a maximum Courant-Friedrichs-Lewy number of 25 is used in all CFD simulations.

\section{Numerical Results of Nonequilibrium Flows}

The purpose of the MPC method is to reproduce, to within 5\%, full DSMC results at a reduced computational expense. This section compares flowfield and surface property results obtained with full DSMC, full CFD, and the MPC method for each of the flow conditions. In addition, code efficiency of the MPC method compared with full DSMC is discussed.

\section{A. Flowfield Properties}

Figure 7 a qualitatively compares the translational temperature contours calculated using full DSMC, full CFD, and the MPC method along with the interface location between the two flow solvers for flow over a cylinder with a global Knudsen number of 0.01. Overall, the MPC method is able to reproduce full DSMC results while limiting the DSMC computation to areas that are rarefied, such as the diffuse shock, boundary layer, and near-wake region. Even in regions of the flow where the continuum-based CFD module is used, the MPC method has improved the agreement with full DSMC over full CFD. This is because the DSMC module has shifted the solution and provides an improved boundary condition to the CFD module. The largest discrepancy between full DSMC and the MPC results, which occurs far away from the body, remains below $4 \%$. Figure $7 \mathrm{~b}$ compares the rotational temperature contours predicted by DSMC, CFD, and the MPC method. Again, agreement is very good, with the largest difference of less than $3 \%$, occurring far away from the body.

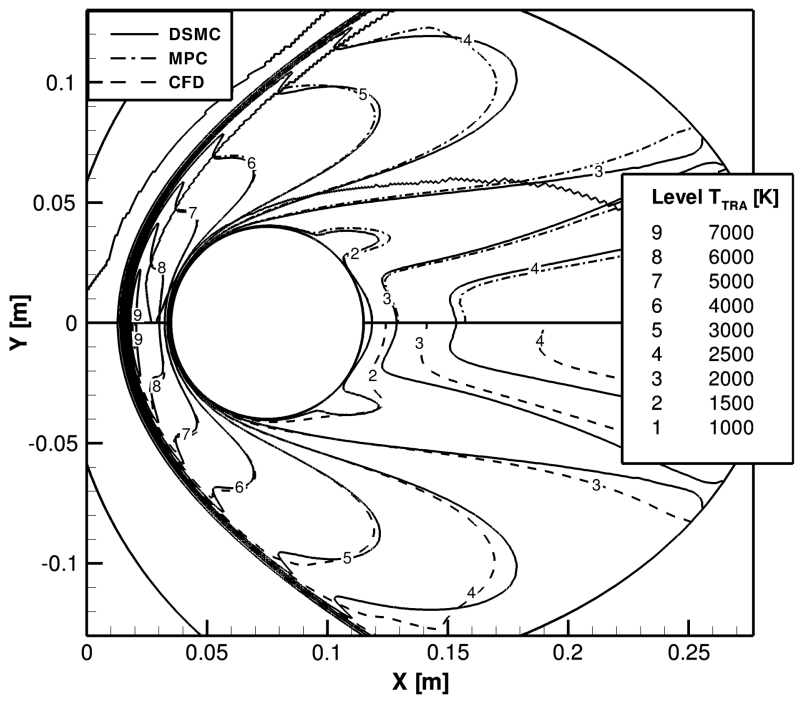

a) $T_{T R A}$

Fig. 7 Comparison of temperature contours predicted by DSMC, CFD, and the MPC method for Mach 12 flow over a cylinder with a global Knudsen number of 0.01 .
Figures $\underline{8 \mathrm{a}}$ and $\underline{8 \mathrm{~b}}$, respectively, compare the translational and rotational temperature contours predicted by DSMC, CFD, and the MPC method for the near-continuum case with a global Knudsen number of 0.002 . The largest discrepancy of $14 \%$ is located in the shock interior, where the CFD module is used despite the flow being highly nonequilibrium. Differences between MPC and DSMC results in the far-wake region, which are located entirely downstream of the body in supersonic flow, remain within $8 \%$ for translational temperature and $5 \%$ for rotational temperature. Previous studies $[12,13]$ and the following subsection show that the differences in the shock structure and far wake have very little effect on the prediction of surface quantities. In addition, the MPC results remain within $2 \%$ of full DSMC results throughout the rest of the shock layer and nearwake region, which directly improve agreement of surface predictions made by the MPC method with full DSMC.

Macroscopic quantities are extracted along two lines (shown in Fig. 5) located at 45 and $135^{\circ}$ from the stagnation streamline. Figure 9 a shows the prediction of translational temperature, rotational temperature, and mass density along a $45^{\circ}$ extraction line for flow with a global Knudsen number of 0.01 . There is very good agreement between full DSMC and the MPC method along the entire extraction line. Especially in highly nonequilibrium regions where large disagreements between full CFD and full DSMC are observed, the difference between MPC and DSMC results is nearly indistinguishable. Even in regions where the CFD module is used, the MPC method has improved agreement with full DSMC compared with the initial full CFD solution. Figure $9 \mathrm{~b}$ shows the comparison of flow variables along the $135^{\circ}$ extraction line, labeled in Fig. 5. In general, full DSMC and CFD are in close agreement same level of agreement. However, near the body, full DSMC and CFD are not in good agreement, while the MPC solution remains in excellent agreement with the full DSMC solution. This improvement near the body has a direct effect on surface property agreement with full DSMC, which will be shown later. Figure 9c compares the variation of macroscopic quantities predicted by $\overline{\mathrm{DSMC}}$, CFD, and the MPC method along a $45^{\circ}$ extraction line for the $K n_{\infty}=0.002$ flow condition. At this flow condition, the shock was simulated using CFD in the MPC simulation. Although there are some disagreements in shock structure with the full DSMC solution, all three methods are in very good agreement from the postshock condition to midway through the thermal boundary layer. Since the CFD module does not accurately model the natural slip at this condition, full DSMC and CFD are not in agreement in the near-wall region. However, the cells very near to the surface are automatically tagged as DSMC cells with the breakdown parameter, and the MPC results are able to reproduce

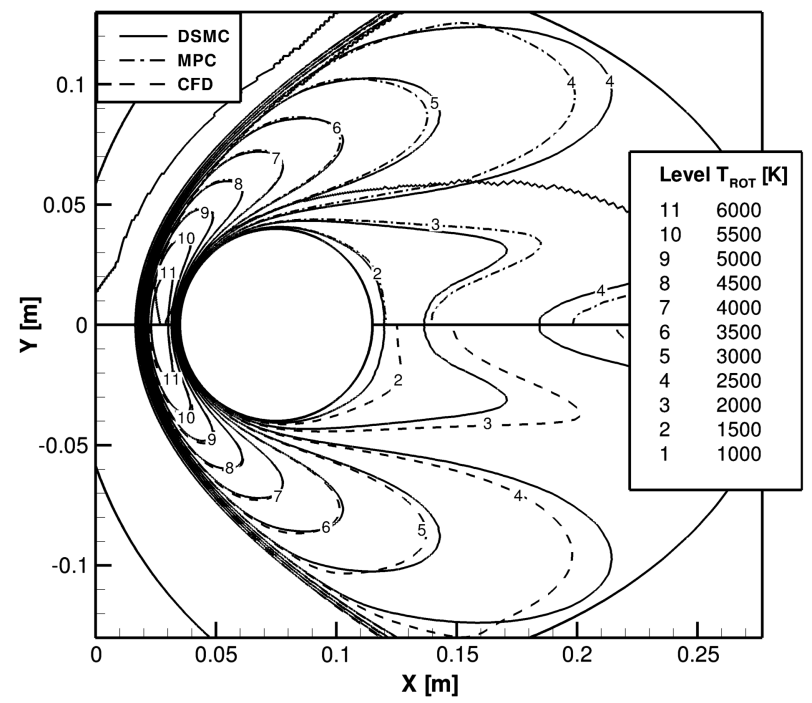

b) $T_{R O T}$ along most of the extraction line, and the MPC method maintains this 


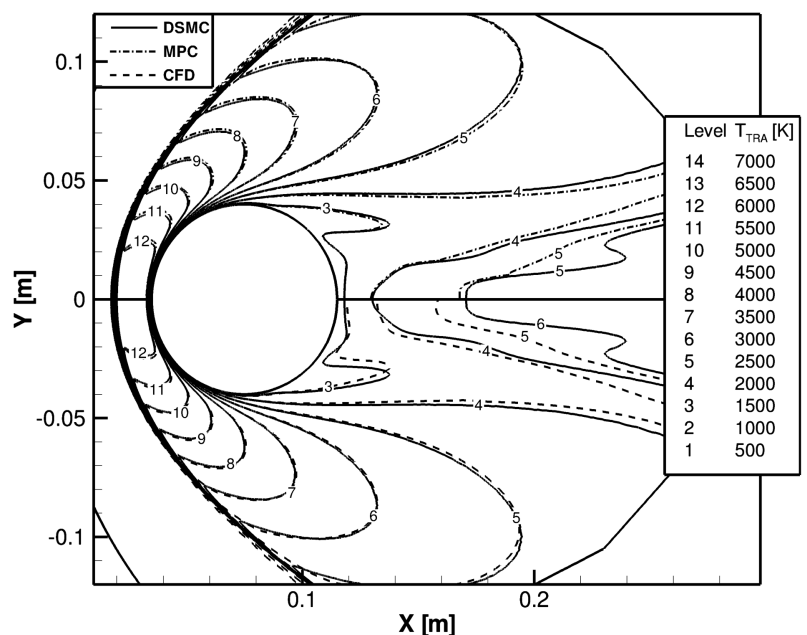

a) $T_{T R A}$

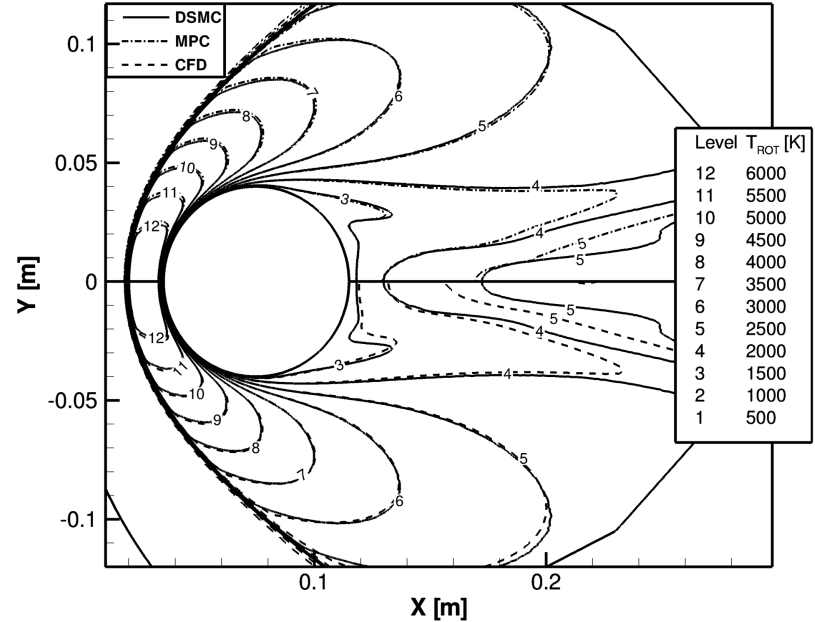

b) $T_{R O T}$

Fig. 8 Comparison of temperature contours predicted by DSMC, CFD, and the MPC for Mach 12 flow over a cylinder with a global Knudsen number of 0.002 .

full DSMC throughout the entire boundary layer. Figure 9d shows the comparison of macroscopic quantities along the $135^{\circ}$ extraction line. As in the higher Knudsen number case, both CFD and DSMC are in very good agreement far away from the body, and the MPC

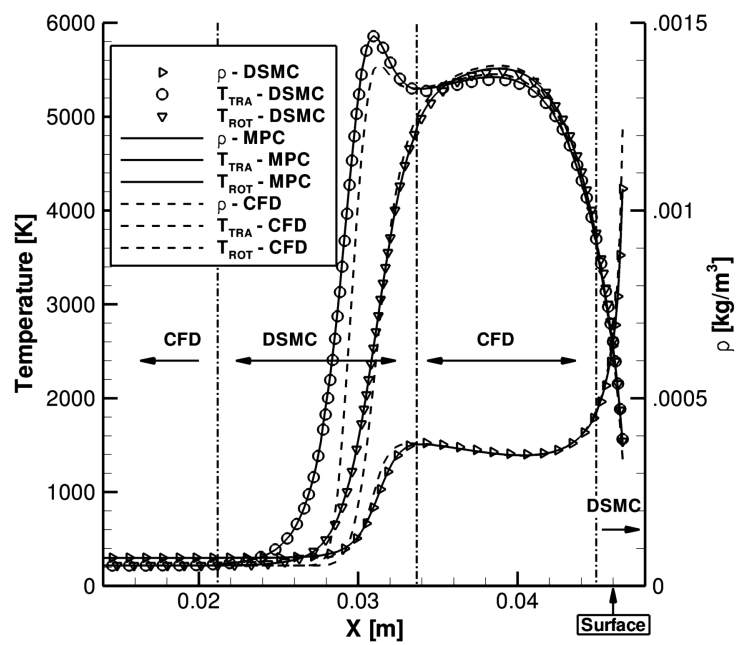

a) $K n_{\infty}=0.01,45^{\circ}$ extraction line

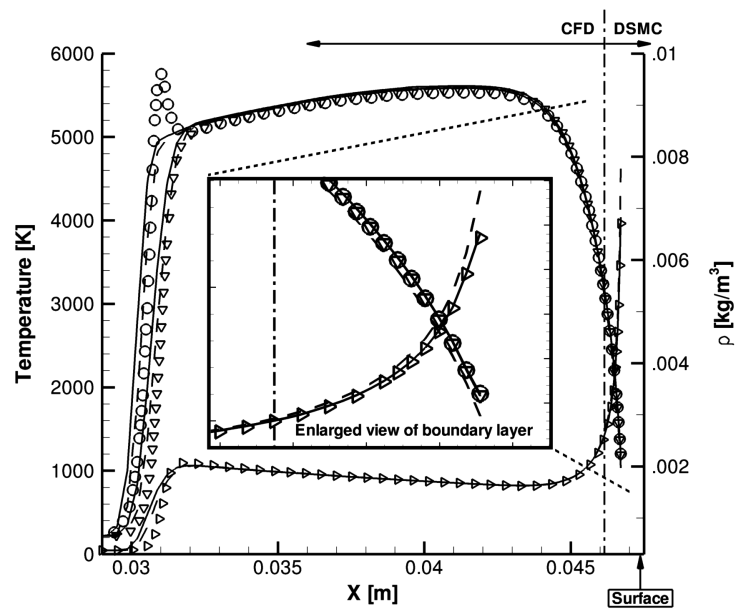

c) $K n_{\infty}=0.002,45^{\circ}$ extraction line method maintains this level agreement. However, near the wall, where the flow is highly nonequilibrium, full DSMC and CFD are in poor agreement, while the MPC method maintains excellent agreement with the full DSMC results.

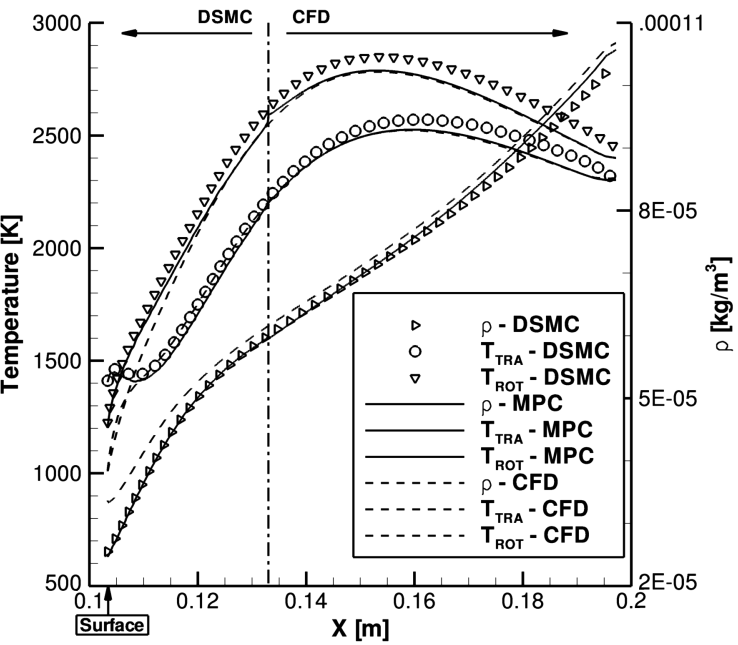

b) $K n_{\infty}=0.01,135^{\circ}$ extraction line

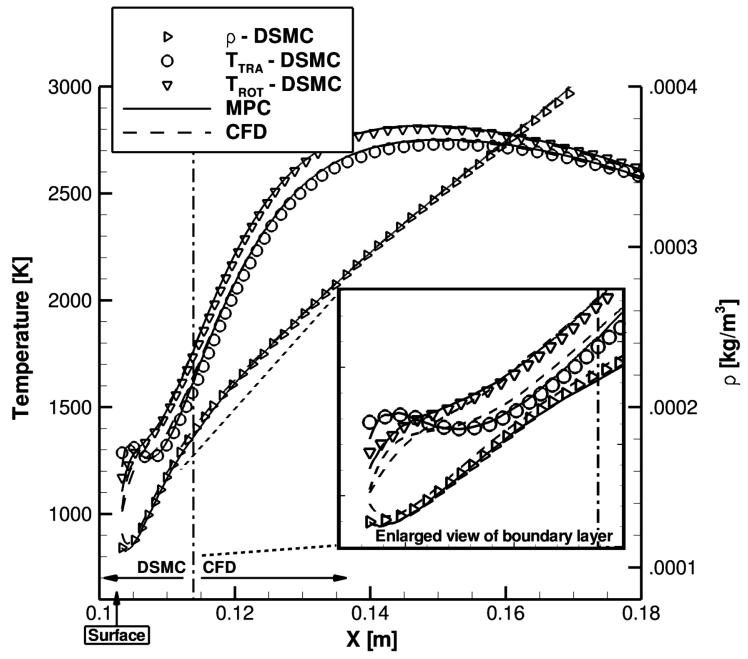

d) $K n_{\infty}=0.002,135^{\circ}$ extraction line

Fig. 9 Comparison along extraction lines of temperatures and density predicted by DSMC, CFD, and the MPC method for Mach 12 flow over a cylinder at two different global Knudsen numbers. 


\section{B. Surface Properties}

For many hypersonic flow problems of interest, accurate prediction of surface properties has a strong impact on vehicle design. Predictions of heat transfer, shear stress, and pressure from full DSMC, full CFD, and the MPC method are compared. Equations (14-16), respectively, show the definitions of the surface pressure, shear stress, and heat transfer coefficient used to compare the surface properties predicted by the simulation methods, where $p$ is the pressure at the wall, $p_{\infty}$ is the freestream pressure, $\tau$ is the shear stress at the wall, $q$ is the heat transfer to the wall, $\rho_{\infty}$ is the freestream density, and $u_{\infty}$ is the freestream velocity:

$$
\begin{aligned}
& C_{p}=\frac{p-p_{\infty}}{(1 / 2) \rho_{\infty} u_{\infty}^{2}} \\
& C_{\tau}=\frac{\tau}{(1 / 2) \rho_{\infty} u_{\infty}^{2}} \\
& C_{h}=\frac{q}{(1 / 2) \rho_{\infty} u_{\infty}^{3}}
\end{aligned}
$$

Figure $10 \mathrm{a}$ compares the surface pressure and heat transfer coefficient on the cylinder surface predicted by DSMC, CFD, and the
MPC method for the $K n_{\infty}=0.01$ case. All three methods remain within $5 \%$ of each other in the forebody region, while the full CFD solution deviates from the full DSMC and MPC results as the flow expands around the cylinder. The largest difference between DSMC and CFD is located in the aftbody of the cylinder, where CFD overpredicts the surface pressure and heat transfer by over $200 \%$. In contrast, the MPC method remains in very good agreement with DSMC along the entire cylinder body. Figure $10 \mathrm{~b}$ compares the shear stress at the surface of the cylinder predicted by DSMC, CFD, and the MPC method for the $K n_{\infty}=0.01$ case. Again, all three methods are in very good agreement along the forebody, but full DSMC and CFD begin to differ as the flow expands around the cylinder, and nonequilibrium effects are large. In addition, the points where the shear stress goes to zero can be used to compare the relative size of the recirculation zone in the near-wake region. Full CFD overpredicts the size of the recirculation zone by $32 \%$ relative to full DSMC, while the MPC method predicts a recirculation zone that differs from full DSMC by $0.7 \%$.

Figure 11a shows the surface pressure and heat transfer coefficients predicted by DSMC, CFD, and the MPC method for the $K n_{\infty}=0.002$ case. For this higher density flow, full CFD remains in agreement with both DSMC and the MPC results along a larger portion of the cylinder but still has large disagreement in the rarefied
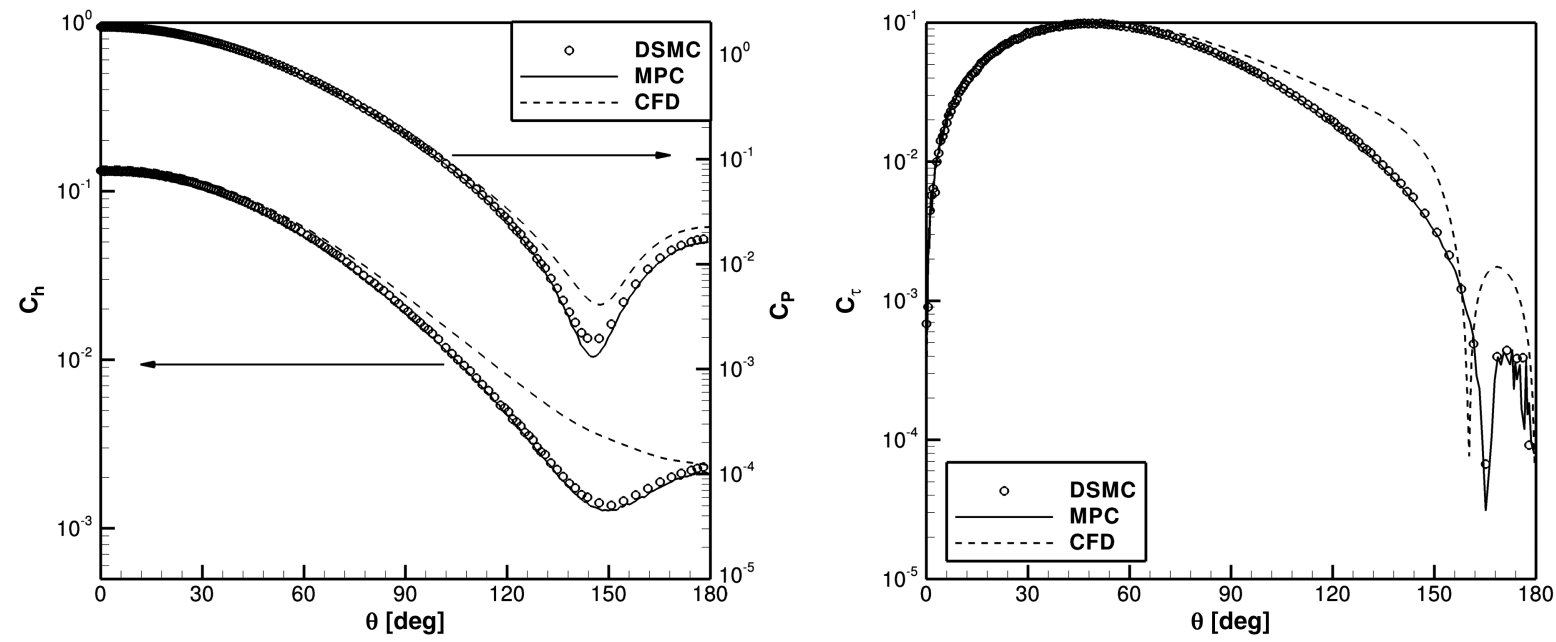

a) $C_{h}, C_{p}$

b) $C_{\tau}$

Fig. 10 Comparison of surface quantities about the cylinder predicted by DSMC, CFD, and the MPC method for Mach 12 flow over a cylinder with a global Knudsen number of 0.01 .

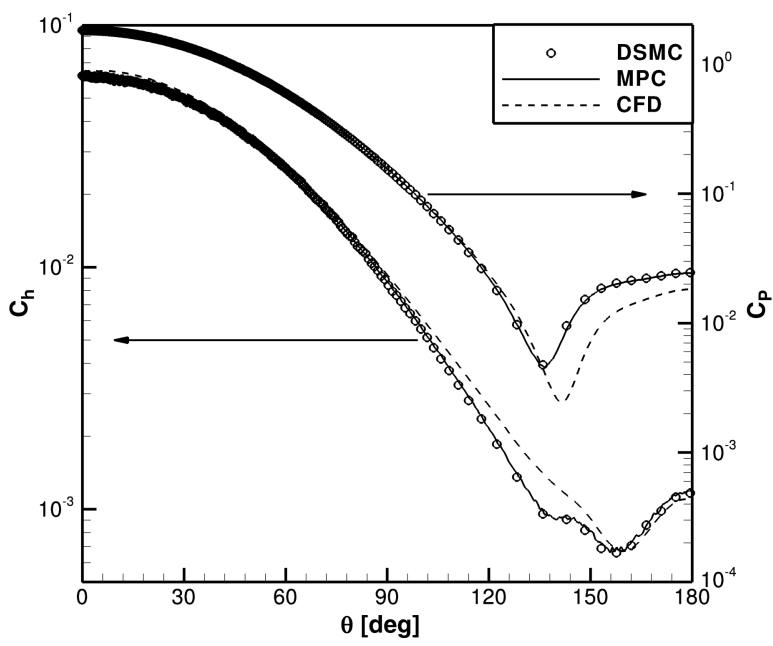

a) $C_{h}, C_{p}$

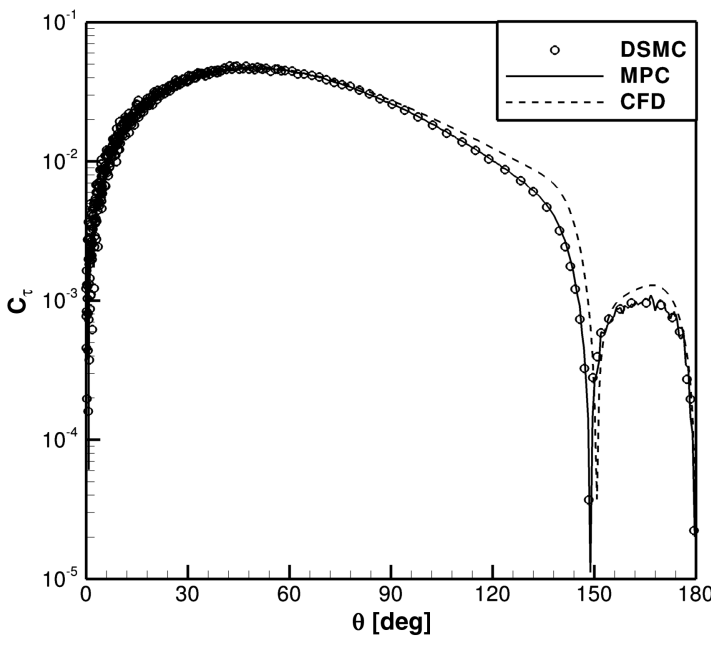

b) $C_{\tau}$

Fig. 11 Comparison of surface quantities about the cylinder predicted by DSMC, CFD, and the MPC method for Mach 12 flow over a cylinder with a global Knudsen number of $\mathbf{0 . 0 0 2}$. 
Table 1 Computational performance and memory requirements for the MPC method

\begin{tabular}{cccc}
\hline \hline$K n$ & Computational cost (speedup) & Ideal speedup & Memory usage \\
\hline 0.01 & $34 . \%(2.94 \times)$ & 2.66 & $28 \%$ \\
0.002 & $3.56 \%(28.1 \times)$ & 17.6 & $75 \%$ \\
\hline \hline
\end{tabular}

wake. Full CFD overpredicts the DSMC heat flux by up to $40 \%$, while it underpredicts full DSMC surface pressure by up to $70 \%$. Interestingly, full CFD slightly underpredicts the surface heat flux along the edge of the recirculation zone, which occurs at angles greater than $150^{\circ}$. This could be due to the underprediction in the size of this recirculation in full CFD relative to the full DSMC result. Again, the MPC method remains in excellent agreement along the entire surface with differences of less than $1.5 \%$ for both surface pressure and heat transfer. Figure $11 \mathrm{~b}$ compares the surface shear stress predicted by DSMC, CFD, and the MPC method. Although the difference between full CFD and DSMC has decreased with the increase in freestream density, there is still some disagreement in the magnitude in the wake region. Interestingly, now, full CFD underpredicts the size of the recirculation zone by over $7 \%$ relative to full DSMC, while the MPC method is within $1.5 \%$ of full DSMC.

\section{Computational Performance}

The MPC method reproduces full DSMC results by using DSMC in rarefied regions while decreasing the computational cost by limiting DSMC to only regions that are in collisional nonequilibrium. Continuum regions are computed using an implicit Navier-Stokes solver. By loosely coupling the methods and studying steady-state flows, the time step used by CFD can be over 100 times larger than the time step required by DSMC for the corresponding flow. In addition, decoupling of the mesh densities allows CFD to be unrestricted by DSMC cell size restrictions, and it can use cells that are up to 18 times larger in area. This significantly decreases the computational time and memory usage required by the MPC method to reproduce full DSMC results. The decreases in computational cost of the MPC method compared with full DSMC are summarized in Table 1. To make a fair comparison, the number of sample time steps after reaching steady state are the same for both full DSMC and the MPC-DSMC module. In addition, all simulations are performed in parallel with the number of processors selected such that the average number of particles on each processor remains constant. Here, the actual speedup is defined as the ratio of the CPU time required for the full DSMC calculation to the CPU time required for the MPC method calculation. The ideal speedup is defined as the ratio of particles used in a full DSMC simulation compared with the number in the corresponding MPC simulation. Since the computational cost of a DSMC simulation nearly scales linearly with the number of particles in the simulation, this should be the upper limit for computational speedup for that problem. The MPC method actually outperforms the ideal speedup ratio. This is because the unsteady portion of the MPC computation is much less than that of the corresponding DSMC simulation, since MPC simulations begin with a fully converged CFD solution. The memory usage is defined as the ratio of the memory used by an MPC simulation to the memory used by the corresponding DSMC simulation. Here, the MPC method requires significantly less memory compared with full DSMC for the higher Knudsen number case and moderately less for the lower Knudsen number case.

\section{Conclusions}

The implementation of rotational relaxation within a MPC method was described and tested on hypersonic blunt-body conditions where regions of the flow exhibited collisional nonequilibrium. An MPC method was outlined that uses existing DSMC and CFD codes with very little modification as modules within a hybrid code. This implementation allows separate updates of either the DSMC or CFD source codes, which reduces the development time of the hybrid code as newer physical models are added to each code. It was found that the MPC method can reproduce full DSMC results at a fraction of the computational time. The rotational models used in both flow modules were described.

In addition, it was found that the empirical gradient-length Knudsen number was not adequate to predict a non-Boltzmann rotational energy distribution function. A separate breakdown parameter that properly tags the flow region where the rotational energy distribution function is highly non-Boltzmann was proposed, tested, and found to be adequate for the flow conditions examined.

Macroscopic flow variables predicted by full DSMC, CFD and the MPC method were compared for two flows with differing global Knudsen numbers. Full CFD and DSMC remained in agreement in regions that were in collisional equilibrium, but they differed significantly in regions of the flow that were highly nonequilibrium. The MPC method remained in very good agreement with full DSMC over the entire flowfield. Surface properties predicted by the three methods were also compared. All three methods remained in excellent agreement for both flow conditions in the highly compressed forebody. However, in the rarefied wake region, the full CFD surface pressure and heat flux differed by over 200 and $40 \%$ compared with full DSMC for the $K n_{\infty}=0.01$ and $K n_{\infty}=0.002$ cases, respectively, while the MPC method remained in excellent agreement, with differences of less than 5\%, with DSMC along the entire surface for both flow cases. In addition, full CFD overpredicted the size of the recirculation zone in the near wake by over 32 and $7 \%$ relative to full DSMC, while the MPC method remained within 0.7 and $1.5 \%$ relative to full DSMC for the $K n_{\infty}=0.01$ and $K n_{\infty}=$ 0.002 cases, respectively.

The computational performance of the MPC method relative to full DSMC was compared for the two flow conditions. By using DSMC only in regions that exhibit nonequilibrium effects, the MPC method was a factor of 2.94 faster than DSMC for the higher Knudsen number case. For the lower Knudsen number case, where a larger portion of the flowfield can be simulated using the CFD module, the MPC method was 28.1 times faster than DSMC.

\section{Acknowledgments}

The authors gratefully acknowledge the financial support provided by NASA grant NCC3-989. The second author acknowledges support under the NASA graduate student researchers program fellowship program (NNX07AV91H) through NASA Ames Research Center, monitored by Michael Wright.

\section{References}

[1] Bird, G. A., Molecular Gas Dynamics and the Direct Simulation of Gas Flows, Clarendon, Oxford, England, U.K., 1994, pp. 218-315.

[2] Glass, C. E., and Gnoffo, P. A., "A 3-D Coupled CFD-DSMC Solution Method With Application to the Mars Sample Return Orbiter," NASA TR TM-2000-210322, July 2000.

[3] Glass, C. E., and Gnoffo, P. A., "Comparison of a 3-D CFD-DSMC Soultion Methodology With a Wind Tunnel Experiment," NASA TR TM-2002-211777, Aug. 2002.

[4] Wilmoth, R. G., Mitcheltree, R. A., Moss, J. N., and Dogra, V. K., "Zonally Decoupled Direct Simulation Monte Carlo Solutions of Hypersonic Blunt-Body Flows," Journal of Spacecraft and Rockets, Vol. 31, No. 6, 1994, pp. 971-979. doi: $10.2514 / 3.26546$

[5] Hash, D. B., and Hassan, H. A., "Assessment of Schemes for Coupling Monte Carlo and Navier-Stokes Solution Methods," Journal of Thermophysics and Heat Transfer, Vol. 10, No. 2, 1996, pp. 242-249. doi: $10.2514 / 3.781$

[6] Hash, D. B., and Hassan, H. A., "A Decoupled DSMC/Navier-Stokes Analysis of a Transitional Flow Experiment," 34th AIAA Aerospace Sciences Meeting and Exhibit, Reno, NV, AIAA Paper 1996-0353, 1996.

[7] Roveda, R., Goldstein, D. B., and Varghese, P. L., "Hybrid Euler/ Particle Approach for Continuum/Rarefied Flows," Journal of Spacecraft and Rockets, Vol. 35, No. 3, 1998, pp. 258-265. doi: $10.2514 / 2.3349$

[8] Roveda, R., Goldstein, D. B., and Varghese, P. L., "Hybrid Euler/Direct Simulation Monte Carlo of Unsteady Slit Flow," Journal of Spacecraft 
and Rockets, Vol. 37, No. 6, 2000, pp. 753-760. doi: $10.2514 / 2.3647$

[9] Wu, J.-S., Lian, Y.-Y., Cheng, G., Koomullil, R., and Tseng, K.-C., "Development and Verification of a Coupled DSMC-NS Scheme Using Unstructured Mesh," Journal of Computational Physics, Vol. 219, No. 2, Dec. 2006, pp. 579-607. doi:10.1016/j.jcp.2006.04.013

[10] Wijesinghe, H. S., and Hadjiconstantinou, N. G., "Discussion of Hybrid Atomistic-Continuum Methods for Multiscale Hydrodynamics," International Journal for Multiscale Computational Engineering, Vol. 2, No. 2, 2004, pp. 189-202. doi:10.1615/IntJMultCompEng.v2.i2.20

[11] Schwartzentruber, T. E., and Boyd, I. D., "A Hybrid ParticleContinuum Method Applied to Shock Waves," Journal of Computational Physics, Vol. 215, No. 2, July 2006, pp. 402-416. doi:10.1016/j.jcp.2005.10.023

[12] Schwartzentruber, T. E., Scalabrin, L. C., and Boyd, I. D., "Hybrid Particle-Continuum Simulations of Nonequilibrium Hypersonic BluntBody Flowfields," Journal of Thermophysics and Heat Transfer, Vol. 22, No. 1, 2008, pp. 29-37. doi:10.2514/1.30216

[13] Schwartzentruber, T. E., Scalabrin, L. C., and Boyd, I. D., "Multiscale Particle-Continuum Simulations of Low Knudsen Number Hypersonic Flow over a Planetary Probe," Journal of Spacecraft and Rockets, Vol. 45, No. 6, 2008, pp. 1196-1206. doi:10.2514/1.37319

[14] Schwartzentruber, T. E., Scalabrin, L. C., and Boyd, I. D., "Modular Implementation of a Hybrid DSMC-NS Algorithm for Hypersonic Non-Equilibrium Flows," AIAA Paper 2007-0613, 2007.

[15] Lian, Y.-Y., Tseng, K.-C., Chen, Y.-S., Wu, M.-Z., Wu, J.-S., and Cheng, G., "An Improved Parallelized Hybrid DSMC-NS Algorithm," AIP Conference Proceedings, Vol. 1084, No. 1, Dec. 2008, pp. 341346. doi:10.1063/1.3076499

[16] Boyd, I. D., "Analysis of Rotational Nonequilibrium in Standing Shock Waves of Nitrogen," AIAA Journal, Vol. 28, No. 11, 1990, pp. 19971999. doi: $10.2514 / 3.10511$

[17] Holman, T. D., and Boyd, I. D., "Numerical Investigation of the Effects of Continuum Breakdown on Hypersonic Vehicle Surface Properties," AIAA Paper 2008-3928, 2008.

[18] Deschenes, T. R., Boyd, I. D., and Schwartzentruber, T. E., "Incorporating Vibrational Excitation in a Hybrid Particle-Continuum Method," AIAA Paper 2008-4106, 2008.

[19] Schwartzentruber, T. E., Scalabrin, L. C., and Boyd, I. D., "A Modular Particle-Continuum Numerical Method for Hypersonic NonEquilibrium Gas Flows," Journal of Computational Physics,
Vol. 225, No. 1, July 2007, pp. 1159-1174.

doi:10.1016/j.jcp.2007.01.022

[20] Scalabrin, L. C., and Boyd, I. D., "Numerical Simulation of Weakly Ionized Hypersonic Flow for Reentry Configurations," San Francisco, CA, AIAA Paper 2006-3773.

[21] Scalabrin, L. C., and Boyd, I. D., "Development of an Unstructured Navier-Stokes Solver for Hypersonic Nonequilibrium Aerothermodynamics," 38th AIAA Thermophysics Conference, Toronto, Canada, AIAA Paper 2005-5203, June 2005.

[22] Dietrich, S., and Boyd, I. D., "Scalar and Parallel Optimized Implementation of the Direct Simulation Monte Carlo Method," Journal of Computational Physics, Vol. 126, No. 2, July 1996, pp. 328 342.

doi:10.1006/jcph.1996.0141

[23] Holman, T. D., and Boyd, I. D., "Effects of Continuum Breakdown on the Surface Properties of a Hypersonic Sphere," Journal of Thermophysics and Heat Transfer, Vol. 23, No. 4, 2009, pp. 660-673. doi:10.2514/1.43509

[24] Parker, J. G., "Rotational and Vibrational Relaxation in Diatomic Gases," Physics of Fluids, Vol. 2, No. 4, July 1959, pp. 449-462. doi: $10.1063 / 1.1724417$

[25] Lumpkin, F. E., III, Haas, B. L., and Boyd, I. D., "Resolution of Differences Between Collision Number Definitions in Particle and Continuum Simulations," Physics of Fluids A, Vol. 3, No. 9, Sept. 1991, pp. 2282-2284. doi: $10.1063 / 1.857964$

[26] Boyd, I. D., Chen, G., and Candler, G. V., "Predicting Failure of the Continuum Fluid Equations in Transitional Hypersonic Flows," Physics of Fluids, Vol. 7, No. 1, Jan. 1995, pp. 210-219. doi: $10.1063 / 1.868720$

[27] Wang, W.-L., and Boyd, I. D., "Predicting Continuum Breakdown in Hypersonic Viscous Flows," Physics of Fluids, Vol. 15, No. 1, Jan. 2003, pp. 91-100. doi:10.1063/1.1524183

[28] Schwartzentruber, T. E., Scalabrin, L. C., and Boyd, I. D., "Investigation of Continuum Breakdown in Hypersonic Flows Using a Hybrid Particle-Continuum Algorithm," AIAA Paper 2008-4108, 2008.

[29] Garcia, A. L., and Alder, B. J., "Generation of the Chapman-Enskog Distribution," Journal of Computational Physics, Vol. 140, No. 1, Feb. 1998, pp. 66-70. doi:10.1006/jcph.1998.5889

[30] Sun, Q., and Boyd, I. D., "Evaluation of Macroscopic Properties in the Direct Simulation Method," Journal of Thermophysics and Heat Transfer, Vol. 19, No. 3, 2005, pp. 329-335. doi: $\underline{10.2514 / 1.12542}$ 